\title{
On the Effect of the Hydrogen Content and Deposition Type on the Grain Nucleation and Grain Growth during Crystallization of a-Si:H Films
}

\author{
A.H. Mahan' ${ }^{1}$, S.P. Ahrenkiel ${ }^{1}$, B.Roy ${ }^{2}$, R.E.I. Schropp ${ }^{3}$, H. Li ${ }^{3}$, and D.S. Ginley ${ }^{1}$ \\ ${ }^{1}$ National Renewable Energy Laboratory, Golden, CO 80401 • ${ }^{2}$ Colorado School of Mines, Golden, CO 80401 \\ ${ }^{3}$ Debye Institute, University of Utrecht, 3508 TA Utrecht, The Netherlands
}

\section{Object(s) of this Study}

-analyze crystallization kinetics for $600^{\circ} \mathrm{C}$ anneal temp.

(1) nucleation rate, (2) grain growth velocity

- determine influence of initial film $C_{H}$ for HWCVD films

- determine influence of deposition type for same film $\mathrm{C}_{\mathrm{H}}$

\section{a-Si:H Film Growth}

HWCVD

$$
\begin{gathered}
\text { Resistive } \\
\text { Heater }
\end{gathered}
$$

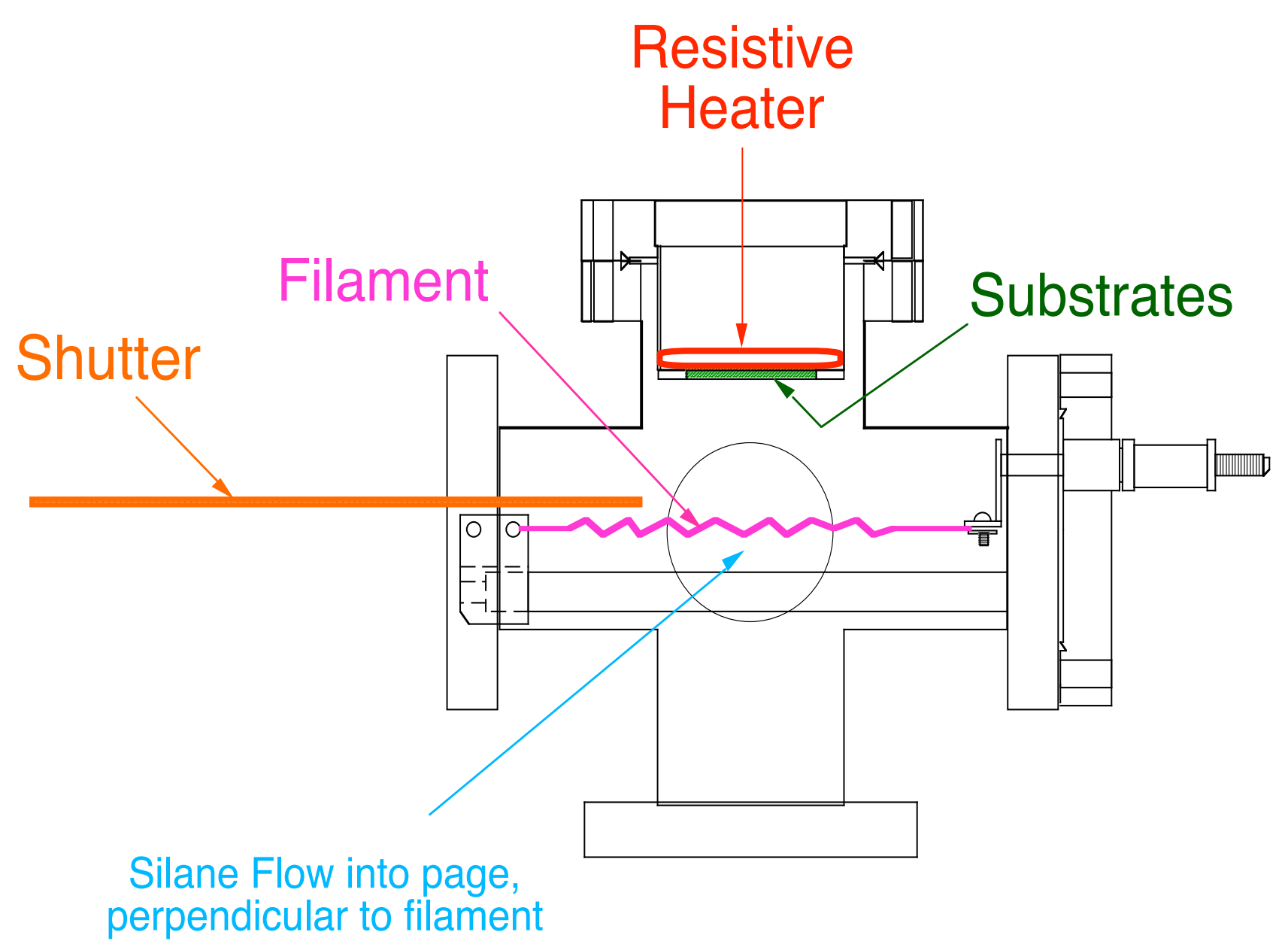

PECVD

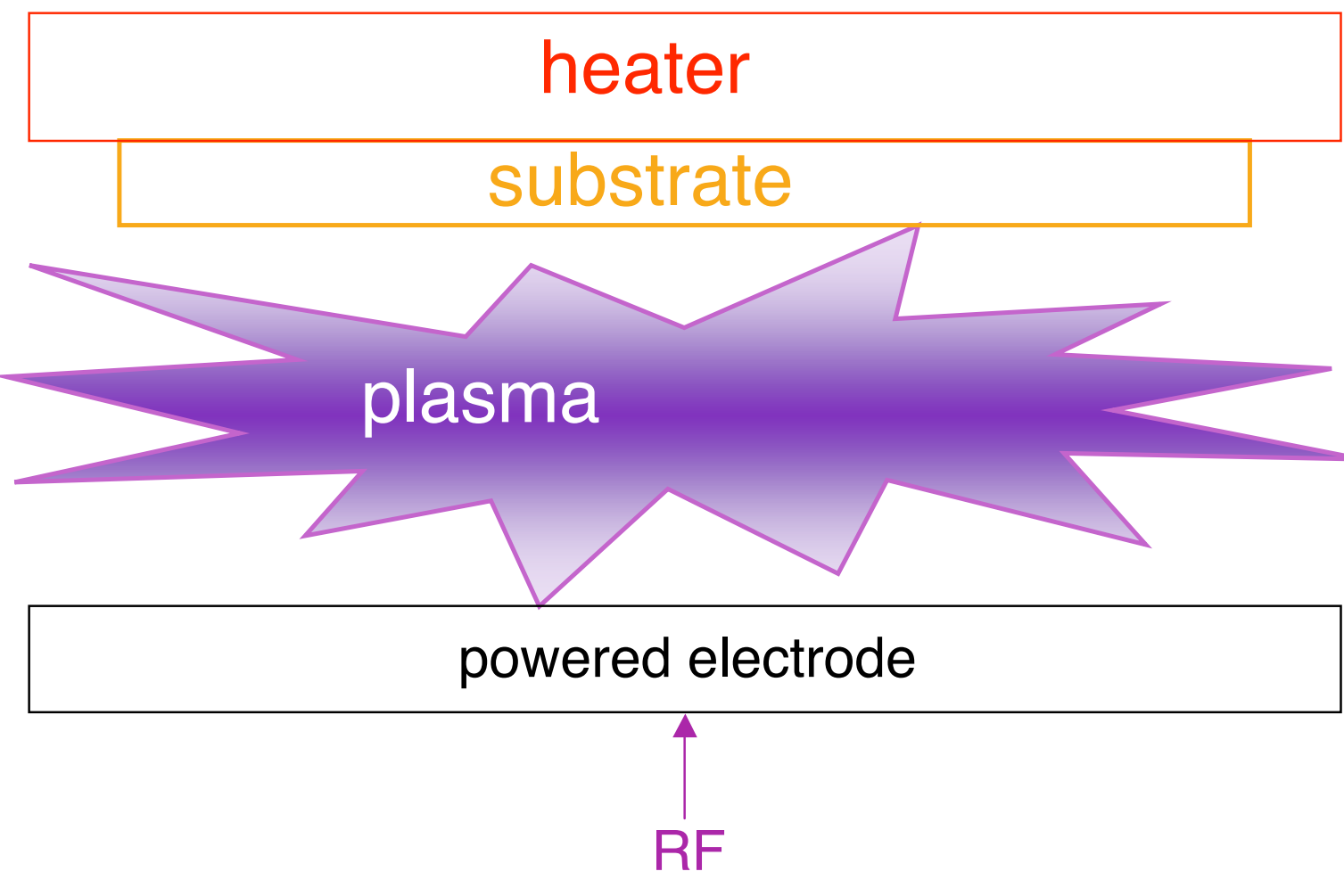

\section{Chacterization Methods}

(1) TEM
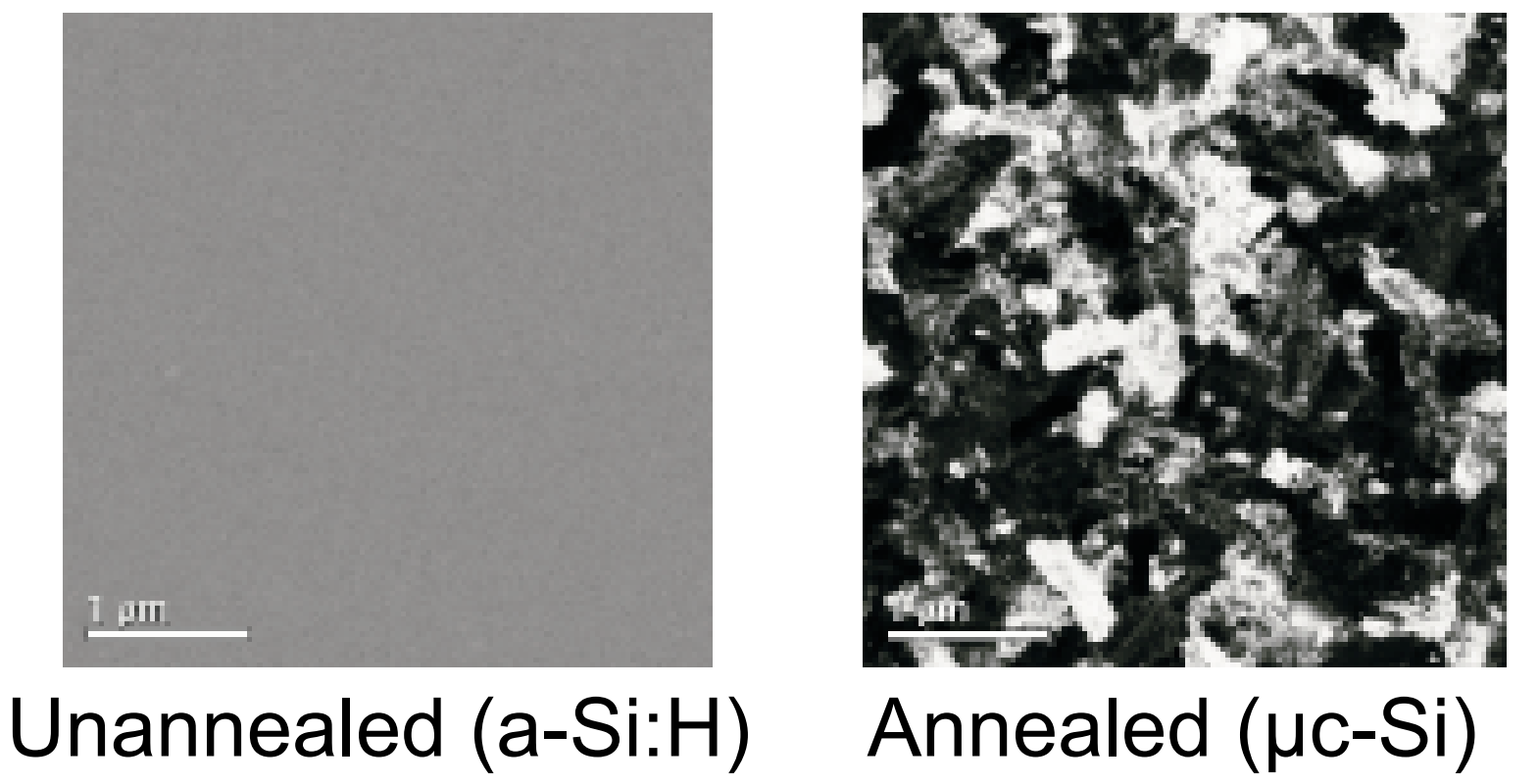

(3) Raman Spectroscopy

(partially annealed, but still amorphous films)

\section{Results}

Measure vs. Anneal Time

- Cryst. Volume fraction

- Grain number density

- XRD (111) peak width

- Raman TO HWHM (a-Si:H)
(2) X-ray Diffraction (XRD)

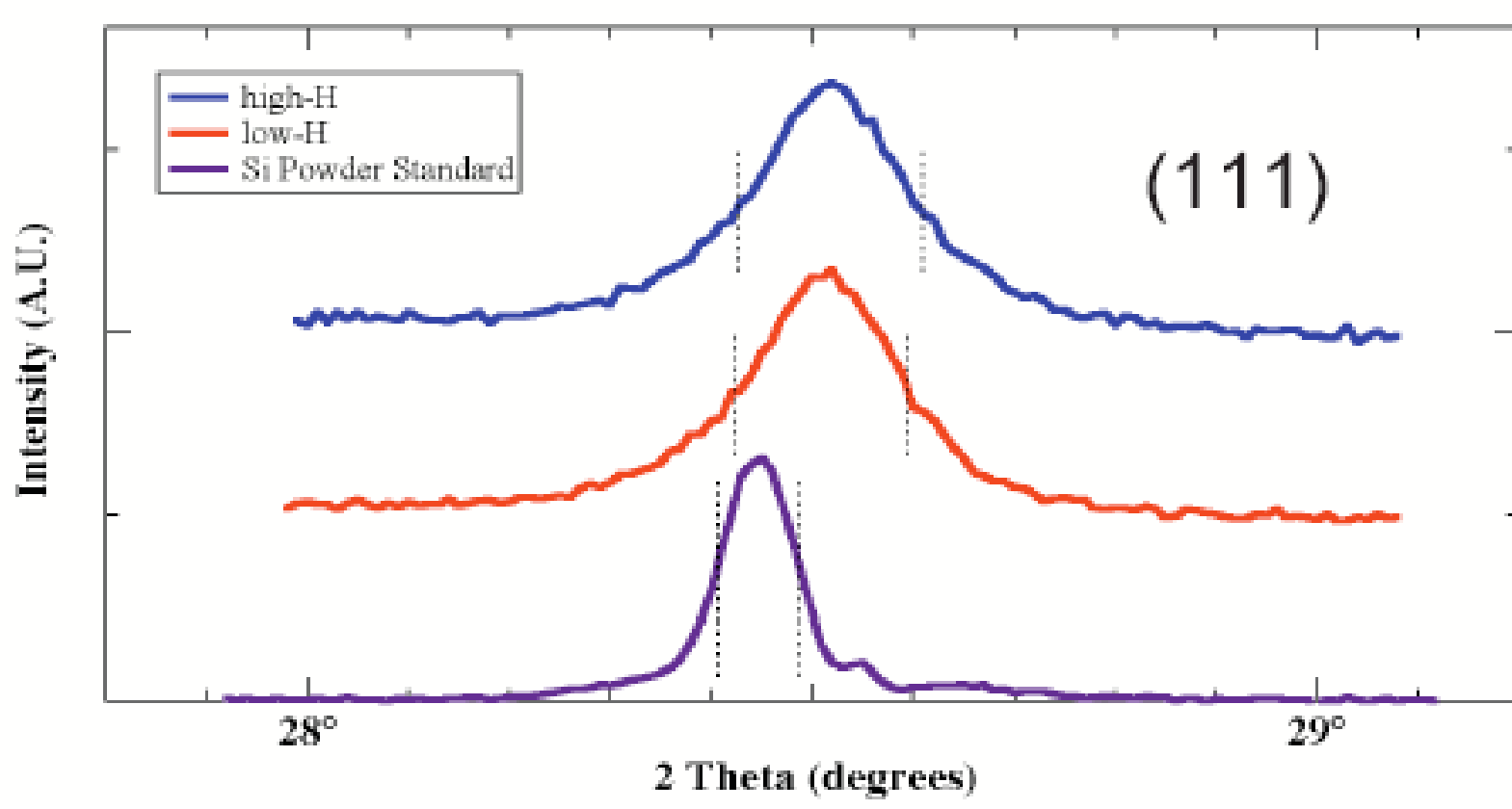

Si (111) peak width comparison)

\section{Calculate}

- nucleation, grain growth rates

- final grain size (TEM)

- XRD 'grain size'

(Scherrer formula)
Does H Evolution Play Any Role in Crystallization? incubation times longer for high $\mathrm{C}_{\mathrm{H}}$ films, much longer for PECVD vs. HWCVD films

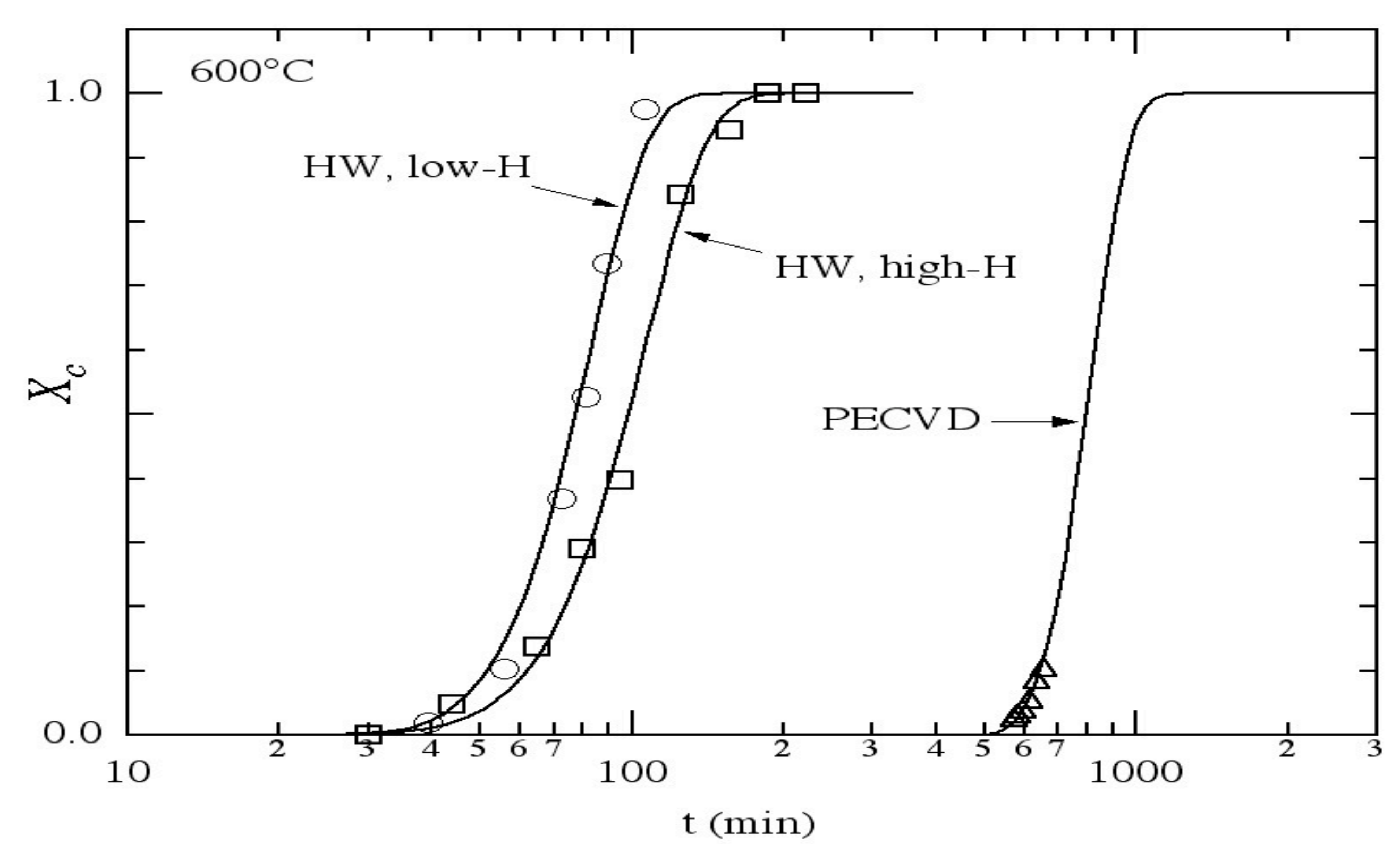

grain growth rates $\sim$ similar for all films - nucleation rates much smaller for PECVD films

\begin{tabular}{|c|c|c|c|}
\hline Film Type & $\begin{array}{c}\text { HWCVD } \\
(3 \text { at. \% H })\end{array}$ & $\begin{array}{c}\text { HWCVD (12 } \\
\text { at. \% H) }\end{array}$ & $\begin{array}{c}\text { PECVD (10 } \\
\text { at. \% H })\end{array}$ \\
\hline $\mathrm{t}_{\mathrm{c}}(\mathrm{min})$ & 62 & 88 & 366 \\
\hline $\mathrm{s}_{\mathrm{g}}(\mathrm{nm} / \mathrm{min})$ & 4.1 & 3.1 & 2.7 \\
\hline $\mathrm{r}_{\mathrm{n}}\left(\mathrm{min} . \mu \mathrm{m}^{3}\right)^{-1}$ & 2.3 & 1.4 & 0.027 \\
\hline
\end{tabular}
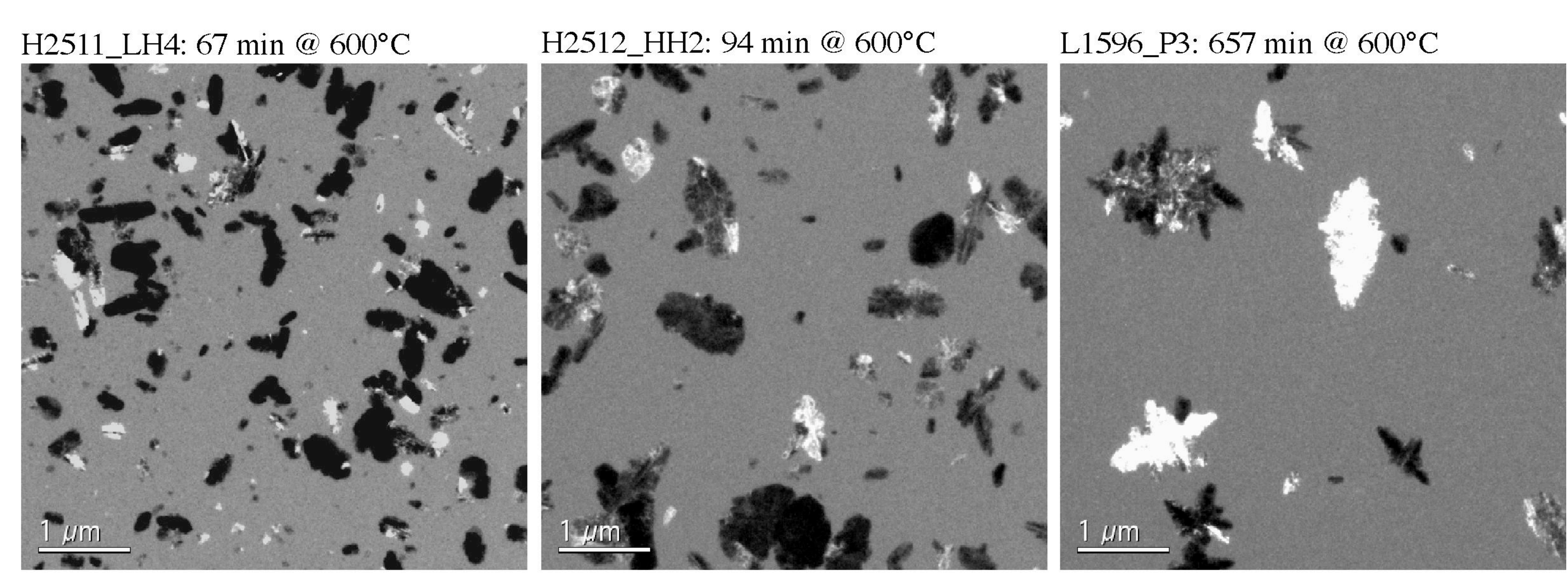

low-H HWCVD

high-H HWCVD

0.34
PECVD same $\mathrm{H}$ content

- Raman TO HWHM increases as $\mathrm{H}$ is evolved from high $\mathrm{C}_{\mathrm{H}} \mathrm{HWCVD}$ film

- high $\mathrm{C}_{\mathrm{H}}$ film also exhibits smaller XRD grain size

\begin{tabular}{|c|c|c|}
\hline Film Type & $\begin{array}{l}\text { HWCVD } \\
\left(\text { low } C_{H} \text { ) }\right.\end{array}$ & $\begin{array}{l}\text { HWCVD } \\
\text { (high } C_{H} \text { ) }\end{array}$ \\
\hline $\begin{array}{l}\text { XRD grain } \\
\text { size }\end{array}$ & $800 \AA$ & $520 \AA$ \\
\hline $\begin{array}{c}\text { Raman } \\
\text { HWHM } \\
\text { (as grown) }\end{array}$ & $28 \mathrm{~cm}^{-1}$ & $28 \mathrm{~cm}^{-1}$ \\
\hline $\begin{array}{c}\text { Raman } \\
\text { HWHM } \\
\text { (annealed) }\end{array}$ & $28 \mathrm{~cm}^{-1}$ & $33 \mathrm{~cm}^{-1}$ \\
\hline
\end{tabular}

- XRD line broadening cannot be due to size effects alone

- significant $\mathrm{H}$ evolution may result in structural disorder (strain, defects)

\section{Conclusions}

- significant difference in incubation times, nucleation rates for HWCVD, PECVD films

- lower PECVD nucleation rates translate into larger grain sizes

- structural disorder caused by $\mathrm{H}$ evolution may affect grain growth 